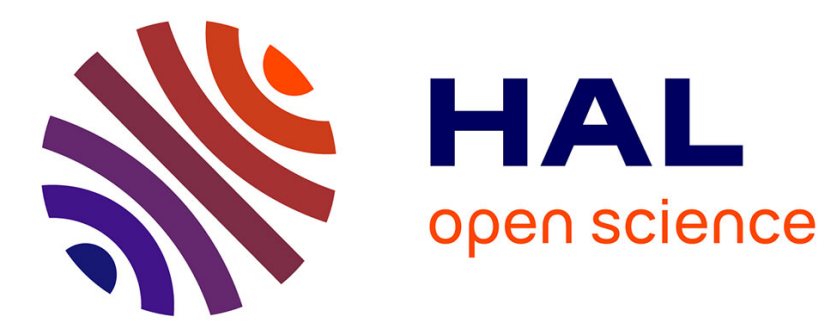

\title{
La géographie humaine en Russie: directions actuelles de recherche et résultats
}

Denis Eckert

\section{To cite this version:}

Denis Eckert. La géographie humaine en Russie: directions actuelles de recherche et résultats. La Revue russe, 1999, 10.3406/russe.1999.2036 . halshs-02566040

\section{HAL Id: halshs-02566040 \\ https://shs.hal.science/halshs-02566040}

Submitted on 6 May 2020

HAL is a multi-disciplinary open access archive for the deposit and dissemination of scientific research documents, whether they are published or not. The documents may come from teaching and research institutions in France or abroad, or from public or private research centers.
L'archive ouverte pluridisciplinaire HAL, est destinée au dépôt et à la diffusion de documents scientifiques de niveau recherche, publiés ou non, émanant des établissements d'enseignement et de recherche français ou étrangers, des laboratoires publics ou privés.

\section{(이) $\$$}

Distributed under a Creative Commons Attribution - NonCommercial - NoDerivatives $\mid 4.0$ 


\section{La géographie humaine en Russie : directions actuelles de} recherche et résultats

Denis Eckert

Citer ce document / Cite this document :

Eckert Denis. La géographie humaine en Russie : directions actuelles de recherche et résultats. In: Revue Russe $\mathrm{n}^{\circ} 16,1999$. pp. 81-94;

doi : https://doi.org/10.3406/russe.1999.2036

https://www.persee.fr/doc/russe_1161-0557_1999_num_16_1_2036

Fichier pdf généré le 30/03/2018 


\section{Denis ECKERT}

\section{La géographie humaine en Russie : directions actuelles de recherche et résultats}

\section{Remarque liminaire}

L'ambition de cet article est de donner une idée de l'évolution récente de la géographie humaine en Russie, sans pour autant prétendre présenter exhaustivement tous les courants ni opérer un dénombrement systématique des publications. Il résulte plutôt du bilan personnel tiré de la présence à des colloques, de contacts et de collaborations entretenues depuis 1991 avec des équipes de recherche en Russie.

\section{Introduction : l'élargissement et le repli.}

Le bilan de la décennie peut être interprété diversement selon les points de vue que l'on adopte. La géographie humaine a d'un côté considérablement profité de la libéralisation de la société, du fait qu'il n'y a plus de sujets de recherche véritablement tabous. De même que les géographes physiciens ont pu aborder des sujets environnementaux (cartographie des pollutions, dégradation des milieux naturels, etc.), les représentants de la géographie humaine ont eu la pleine liberté d'analyser sous toutes ses coutures les transformations de l'organisation territoriale de la société (phénomènes de migrations internes et externes, devenir des périphéries, modalités régionales de la transition économique), y compris dans leurs aspects les plus conflictuels : géographie des crises ethniques, apparition de frontières. Le symbole le plus fort de cette liberté acquise au cours de la perestroïka a été le développement des recherches sur le renouveau religieux (cartographie de la pratique religieuse, des nouveaux mouvements spirituels...) et la mise en évidence des problèmes sociaux (délinquance, alcoolisme).

Du fait de cette liberté d'analyse, la géographie humaine a d'ailleurs considérablement renforcé ses positions par rapport à la géographie physique naguère hégémonique. Elle est plébiscitée par un nombre croissant d'étudiants, qui se pressent aux inscriptions des chaires correspondantes. Les thèmes qui préoccupent les géographes «humains" sont en prise directe sur les transformations sociales, géopolitiques et économiques. Tout cela donne à la discipline une flatteuse réputation d'utilité sociale.

Denis Eckert est géographe, chargé de recherche au CNRS (Centre Interdisciplinaire d'Études Urbaines de Toulouse, 5 allées A. Machado, 31058 Toulouse Cedex).

mel: eckert@univ-tlse2.fr

LA REVUE RUSSE, Paris, 16, 1999, p. 81-93. 
Mais, d'un autre côté, la portée géographique des champs de recherche s'est réduite. Les géographes russes n'ont plus la possibilité de lancer de vastes projets internationaux, de se spécialiser dans l'étude d'aires géographiques lointaines. Bien des spécialistes qui il y a une décennie travaillaient, dans le cadre des chaires d'études internationales ("des pays capitalistes", « des pays socialistes ») sur des espaces lointains, ont replié leur recherche sur la Russie même et ses plus proches voisins. Il leur est impossible de trouver en Russie des financements pour des missions de terrain à l'étranger. Le salut aurait pu venir des institutions et fondations étrangères (INTAS, Carnegie, McArthur, Humboldt, CNRS, etc.) qui ont aujourd'hui un rôle très important dans le soutien à la recherche : mais elles financent prioritairement, dans le domaine des sciences humaines, des projets ayant trait à l'évolution de la Russie même ; elles n'incitent guère les chercheurs russes à analyser ce qui se passe ailleurs. La contribution demandée aux scientifiques russes n'est pas non plus de nature théorique : il ne leur est pas demandé de contribuer à la construction de nouveaux schèmes interprétatifs globaux, de nouveaux paradigmes universels. On leur assigne plutôt leur champ d'étude « natif » et on les subventionne pour cela. L'auteur de ces lignes participe d'ailleurs lui-même à ce processus de financement d'équipes de recherche russes sur des projets concernant la seule Russie.

D'ailleurs, même à l'intérieur de l'ancienne Union soviétique, les champs de recherche ont été soumis à rétrécissement. L'apparition à la place de l'U.R.S.S. de quinze États indépendants a provoqué un renchérissement considérable des déplacements, et l'éclatement des systèmes statistiques, qui évoluent séparément dans chaque État, rend de plus en plus problématique les recherches portant sur l'espace de l'ex-U.R.S.S. (processus migratoires, par exemple).

En Russie proprement dite, les possibilités de rencontre entre chercheurs de différentes régions sont devenues très rares. Il est paradoxalement plus facile pour un chercheur actif de trouver une bourse pour aller effectuer un séjour de recherche dans une institution étrangère, à Paris, Birmingham, Boston ou Berlin que d'avoir la possibilité de participer à un colloque dans une grande ville de la province russe. L'élévation du prix des billets de train et d'avion ralentit considérablement les échanges scientifiques à l'intérieur même du pays. Dans ces conditions difficiles, une association professionnelle comme l'Académie Internationale du Développement Régional a bien du mérite à organiser, vaille que vaille, un colloque national chaque année, toujours dans une ville différente (1997: Nijni Novgorod, 1998 : Togliatti, 1999 : Iaroslavl), sous la menace permanente de ne pas obtenir à la dernière minute la maigre mais indispensable subvention de la Fondation Russe de la Recherche Fondamentale ${ }^{1}$.

\section{Champs en ébullition}

L'un des aspects les plus remarquables de la géographie humaine actuelle est sa capacité à s'adapter aux mouvements de la société et du pays, à lancer de nouvelles pistes de recherche. Faute de pouvoir faire un tableau complet de la situation, je décrirai quelques champs remarquables. 


\subsection{La géographie électorale}

L'apparition de consultations régulières aux niveaux fédéral, régional, municipal a provoqué une floraison d'études. Les géographes politiques ont ainsi très fortement contribué aux recherches sur la démocratisation de la société. Ils ont mis en évidence des structures spatiales fortes, repérables d'élections en élections, comme la «ceinture rouge » (Kolossov, Platé 1997). La stabilité de cette géographie des sensibilités politiques sur plusieurs années (on a un recul de presque dix ans aujourd'hui) est particulièrement remarquable dans un pays qui n'avait aucune tradition démocratique, et où l'on pouvait croire que la confusion des premières années provoquerait des changements incessants d'orientation politique de l'électorat. Les géographes ont particulièrement contribué à l'explication de l'orientation politique des régions, insistant sur le fait que le couple rural-urbain, pas plus que la prospérité des régions, ne suffisaient à rendre compte intégralement de l'opposition entre vote conservateur («patriotique » et néocommuniste) et vote libéral. Ils ont su montrer également l'influence des traditions sociales anciennes, pointant le fait que le vote des zones ayant jadis connu la forme la plus dure du servage est plus conservateur que celles d'ancienne franchise paysanne (Kolossov, in Eckert, Kolossov 1999).

Aujourd'hui, la cartographie des résultats s'affine : certaines études, menées à l'échelle du bureau de vote, ont montré comment le paysage électoral de la ville de Moscou est corrélé aux différences d'habitat. Le travail de publication des résultats des consultations fédérales effectué par la Commission Electorale Centrale (tout est disponible sur cédérom au niveau géographique le plus fin) permet aujourd'hui d'envisager des études novatrices : on peut traiter les résultats au niveau de toutes les villes et tous les districts de Russie, sortir donc du cadre contraignant de la cartographie électorale par 89 régions (oblasts et républiques).

Les géographes politiques ont profité du large intérêt porté aux élections; ils ont publié leurs résultats en dehors du cercle des revues géographiques: notamment dans la revue Polis; leurs travaux sont d'ailleurs connus et appréciés à l'étranger.

\subsection{La géographie sociale et urbaine}

La géographie urbaine a su coller aux transformations de l'espace des villes. Des études montrent comment sont apparues les nouvelles fonctions (commerce de luxe, activités de services de haut niveau) et comment l'espace résidentiel de Moscou s'est modifié. Dans le cas de la capitale, où le marché foncier s'est structuré très rapidement à partir de 1992, les données sur le prix moyen des transactions immobilières par quartier ont permis de mettre en évidence la formation des quartiers " chics », beaucoup moins homogènes néanmoins qu'en Europe occidentale. Cette nouvelle géographie sociale de Moscou est très largement l'héritière des quartiers « de prestige » de l'époque soviétique : Centre, Sud-Ouest (Collectif 1999).

Les transformations très rapides de l'espace urbain ont aussi stimulé des études qui, à la frontière de la géographie et de l'urbanisme, montrent sur 
quelles bases idéologiques et avec quels moyens les municipalités cherchent à encadrer le changement de leurs villes.

\subsection{La géopolitique}

Les études géopolitiques sont un autre champ où les géographes russes ont été particulièrement productifs. Il est vrai que le contexte agité de la disparition de l'U.R.S.S., la formation de nouveaux États, les contestations nombreuses de frontières, l'ébullition des nationalismes et les conflits qui ont secoué l'espace post-soviétique constituaient un terrain privilégié d'étude. Toutes les situations de crise et de guerre (Abkhazie, Tchétchénie, Transnistrie) ont été attentivement étudiées et passionnément commentées. On a beaucoup travaillć sur les risques, cherchant à prévoir les cas de figure où la probabilité de naissance d'un conflit ethnique était la plus élevée. Mais les recherches concernent aussi des phénomènes plus paisibles, comme l'apparition de frontières d'État et ses conséquences (par exemple entre Ukraine et Russie).

La question des minorités ethniques est suivie constamment. Les conséquences politiques de l'émergence d'un nationalisme ethnique dans des pays aux populations très mêlées ont suscité un intérêt soutenu. Les flux migratoires des dernières années, notamment ceux des réfugiés, risquent-ils de modifier l'identité des régions, de provoquer une menace sur l'intégrité du territoire ? La question se pose surtout dans le Nord-Caucase, où, aux marges du Daghestan et de la Tchétchénie, des flux migratoires modifient considérablement la donne ethnique. La question qu'on se pose, à l'arrièreplan de toutes ces études, est bien sûr celle de la capacité de la Russie à maintenir l'unité de son espace malgré la montée des nationalismes locaux et des pouvoirs régionaux, et à garder sa place dans le monde ou par rapport à ses voisins.

Les spécialistes de géopolitique cherchent à conceptualiser la position de leur pays par rapport au reste du monde : est-ce

- une marge de l'Europe, toujours en retard sur le train de l'innovation et du changement ?

- le centre d'une Eurasie ayant pour contours la CEI ?

- l'un des éléments structurants d'une grande Europe en lente intégration?

Pour beaucoup de spécialistes, la publication de l'ouvrage de Samuel Huntington Le Choc des civilisations a été un événement traumatique (1997). Cet universitaire américain a popularisé des conceptions de médiocre géopolitique selon lesquelles les frontières religieuses millénaires qui traversent l'Europe (chrétienté occidentale / orthodoxie, chrétienté / islam) sont des lignes de fracture actives entre modèles de société radicalement opposés. Il explique ainsi tout le climat politique actuel en Europe, réifiant des frontières religieuses promues au rang de fatalités historiques indépassables. Il s'agit dans sa pensée quasiment de frontières «naturelles » entre des civilisations opposées et évacue toute évolution historique des sociétés. La position géopolitique du pays est donc évaluée sur la base de structures « culturelles » intangibles, alimentant une pensée fixiste où l'analyse de la 
dynamique des situations n'a pas de place. Cette thèse dont de nombreux aspects sont particulièrement pernicieux a eu un retentissement extraordinaire en Russie. Presque toute l'intelligentsia et une partie de la classe politique se sont emparés de cet ouvrage, pour y trouver la preuve que l'Occident est structurellement hostile à la Russie. On y trouve d'ailleurs une commode justification au repli sur soi : puisque ces frontières sont à jamais inscrites sur les cartes... Huntington permet fort commodément de ne pas s'interroger sur les causes internes du mal-développement économique et social du pays.

\subsection{Un champ traditionnel toujours actif : la géographie du peuplement}

Point fort de la géographie humaine en Russie, la géographie du peuplement continue de susciter un grand intérêt. La Russie reste en effet fidèle à sa tradition de pays en mouvement perpétuel. Depuis quelques années, les directions des flux migratoires se sont modifiées du tout au tout. Les auteurs ont su, face aux mouvements de repli vers la Russie de nombreux migrants venus de toutes les périphéries soviétiques, en montrer le caractère relativement ancien. La disparition de l'U.R.S.S. n'a fait qu'accélérer des mouvements de long terme : on pense aux migrations de russophones en provenance de Transcaucasie, qui ont débuté dans les années 60 . Le déclin démographique des périphéries de la Russie proprement dite est aussi suivi avec attention (retours de l'Extrême-Orient, crise démographique du Grand Nord). De même, les provinces qui attirent actuellement les migrants (dans le Sud, notamment) ont fait l'objet d'enquêtes de terrain poussées.

On avait cru discerner, dans les premières années de la crise, un mouvement de reruralisation du pays : les villes, disait-on, étaient en train de perdre une part significative de leur population, qui allait chercher en ces temps difficiles de quoi survivre à la campagne. Les chercheurs les plus en vue ont su montrer que cette tendance était largement illusoire. Car si effectivement, en marge des agglomérations importantes, on a vu s'installer des citadins paupérisés, la campagne profonde a continué à se dépeupler : dans la plupart des régions, la dégradation de l'accessibilité des villages périphériques (destruction progressive des routes, faute d'entretien) accélère considérablement la désertification. Les hommes se concentrent plus qu'avant autour des centres urbains. Le géographe B. Rodoman soutient que les processus actuels ont conduit à la production d'un territoire éclaté. Il existe désormais selon lui trois Russies :

- toutes les zones situées à moins de deux heures des aéroports internationaux, c'est-à-dire un archipel de grandes régions urbaines ou exportatrices, ouvert sur le monde, sensible aux variations du dollar, où l'on achète voitures et électroménager importés. Ses habitants se considèrent volontiers comme des Européens comme les autres ;

- une Russie provinciale se débrouillant par elle-même, celles des villes petites et moyennes, souffrant de la crise de l'emploi et des retards de salaire, qui dessine un univers sans guère de perspectives où les horizons sont moins larges et les mentalités plus frileuses ;

- la Russie de la périphérie profonde, qui commence à deux kilomètres des dernières routes goudronnées et est coupée de presque toute forme 
moderne de consommation. Ses habitants vivent de peu. Cette Russie des marges s'étend sur dix millions de kilomètres carrés (vingt fois la France !)

Malgré la vigueur des recherches, les analyses des spécialistes de la géographie du peuplement souffrent de l'absence de grands outils de statistique territoriale qui, au niveau fédéral, permettraient de suivre et modéliser la dynamique du peuplement (voir infra 4.2).

\subsection{La géographie économique entre tradition et analyse du changement.}

La géographie économique soviétique était naguère une discipline puissante, dont les représentants étaient associés, pour le meilleur mais aussi pour le pire, à la préparation des décisions d'aménagement des autorités ${ }^{2}$. Aujourd'hui, face à un espace économique en mutation rapide, elle peine à produire des schèmes interprétatifs globaux et ne réussit pas à proposer des modèles convaincants de développement régional.

Ceci s'explique en partie par la confusion actuelle : les données économiques sont de celles qui sont le plus délicates à aborder, tant la significativité des indicateurs est douteuse (voir infra 4.1). Mais au-delà de ce contexte très difficile, l'influence de l'arrière-garde mandarinale, aux conceptions très traditionnelles ${ }^{3}$, ne contribue pas à renouveler les problématiques et à aérer la bibliothèque théorique. Certains spécialistes continuent de s'accrocher à la théorie de la base industrielle de l'urbanisation : les villes ne peuvent naître et croître, dans ce schéma, qu'en liaison étroite avec le développement industriel. Ces thèses apparaissent comme dépassées, d'une part parce que le contexte russe est celui d'une récession particulièrement sévère - les dynamiques respectives des villes et de l'industrie sont donc découplées -, d'autre part parce que l'intérêt des analystes, de par le monde, se tourne de plus en plus vers le fonctionnement spatial des économies postindustrielles.

Les prises de position politiques jouent aussi leur rôle. Les conservateurs, dont on entend périodiquement les prises de position dans les colloques et les séminaires, peuvent proclamer par exemple que la fermeture des frontières permettrait certainement de restaurer les échanges régionaux et les « équilibres » antérieurs à la désétatisation de l'économie.

D'autres se font les apôtres d'une géographie du potentiel. Ils cherchent à évaluer les capacités des différentes parties du pays à enclencher un processus de croissance. Ils s'acharnent à définir des "climats régionaux d'investissement », des «potentiels de développement », sous forme de typologies statistiques des régions de Russie. Ces typologies sont basées sur la compilation de statistiques régionales, à partir desquelles on élabore ensuite une formule synthétique : un zeste de taux de chômage, trois cuillères à soupe de taux d'urbanisation, deux rasades de valeur ajoutée industrielle, etc., et l'on obtient une note pour chacune des régions russes. Ces travaux font la part belle à la statistique économique, pourtant en grave crise : on souhaiterait, dans le contexte brouillé de l'information statistique russe, qu'on utilise avec plus de prudence et de modération les dizaines et dizaines d'indicateurs moulinés dans ces typologies. Le niveau de subtilité de tels traitements statistiques est d'ailleurs très faible : l'information est, d'un 
indicateur à l'autre, très souvent redondante. S'agit-il d'un désir d'enivrement par des hectolitres de statistiques, qu'elle qu'en soit la validité, afin de passer sous silence une réalité moins rose ? On retrouverait là un réflexe bien soviétique.

Ces travaux font l'impasse sur la question de l'accessibilité, dont j'estime personnellement qu'elle est au centre des problématiques de développement des régions russes, (comment assigner à une région un fort potentiel de développement touristique, à mille lieues de toute clientèle et en l'absence d'infrastructures d'accueil ?), mais leurs auteurs dédaignent de surcroît de replacer leurs classements dans un cadre international plus large : faire une géographie du potentiel d'investissement des régions sans prendre en compte le fait que les investisseurs raisonnent à l'échelle de la planète revient à ne pas se poser la question des avantages comparatifs de ces régions dans le système économique mondial. C'est une géographie du rêve, qui s'éloigne de ce fait de toute dimension pratique, de tout transfert. Les investisseurs de chair et d'os sont eux bien indifférents face à de tels travaux. Leur évaluation des aménités de telle ou telle région est plus pragmatique. Une enquête menée dans le cadre d'un programme de recherche international ${ }^{4}$ fait apparaître que les hommes d'affaires étrangers n'accordent pratiquement aucun crédit à ces savantes typologies, et mettent en avant soit leur propre logique de secteur (il faut aller là où sont les partenaires potentiels) soit choisissent systématiquement les régions les plus accessibles pour effectuer un choix d'investissement.

On ne saurait réduire les résultats de la géographie économique russe à ces piètres productions. Certains résultats incontestables, venant de la part d'observateurs moins dogmatiques, montrent notamment :

- la forte accentuation, en même temps que s'effectuait la transition vers l'économie de marché, des différences entre régions, que ce soit en terme de revenu des habitants, de consommation, de valeur ajoutée. Ceci au point que la Russie serait l'un des pays au monde où ces différences sont les plus marquées : n'y a-t-il pas là menace sur la cohésion sociale et politique de tout l'espace russe ? Un handicap rédhibitoire au fonctionnement d'un véritable fédéralisme ? Pourtant, à un moment où on parle beaucoup, au niveau politique, de la prédominance des tendances centrifuges, de la constitution de macro-régions politiques, on a pu montrer que la crise n'avait pas provoqué l'apparition de grands marchés régionaux indépendants les uns des autres (une Sibérie occidentale plus ou moins autonome, par exemple). Les régions contiguës n'ont pas tendance à échanger entre elles davantage qu'avec le reste du pays ;

- la concentration de la valeur ajoutée industrielle dans quelques régions (Iamal-Volga-Oural), marquant ainsi une contraction de l'espace productif. En dehors de ces quelques forteresses, la géographie de l'industrie doit être faite à une échelle fine, car à l'intérieur même d'une région, d'une petite ville à l'autre, les situations peuvent être radicalement différentes (Treivish 1998);

- le renforcement du rôle de Moscou, leader économique qui a la fois concentre les richesses mais centralise le pouvoir de décision dans tous les 
domaines (banque, industrie, services de haut niveau). Ce centralisme économique, plus fort qu'à l'époque soviétique, résulte d'une politique de rachat systématique des meilleures entreprises provinciales par les hommes d'affaires de la capitale. Ce processus est en partie freiné depuis la crise d'août 1998, du fait de la faillite de nombreux groupes financiers moscovites.

\section{Lire et publier}

Les géographes sont confrontés comme tant d'autres disciplines aux conséquences néfastes de la crise structurelle du monde académique. Alors même que les champs de recherche possibles s'élargissent, le manque de moyens provoque un certain isolement intellectuel et entrave la diffusion des résultats obtenus.

\subsection{L'accès aux ressources documentaires}

La vie intellectuelle souffre fortement de la réduction à presque rien des moyens des bibliothèques. À l'époque soviétique, les chercheurs de l'Académie des Sciences avaient facilement accès à toutes les revues internationales de référence en anglais, français, allemand et étaient fort bien informés de l'évolution générale de leur discipline. Les bibliothèques de recherche avaient la possibilité de souscrire de nombreux abonnements à des publications étrangères. Les abonnements internationaux ont été presque tous supprimés, d'autant plus vite que les tarifs des revues anglophones sont en général prohibitifs. Certaines publications sont toujours reçues ${ }^{5}$, mais en général le contact avec les publications étrangères se fait par le biais de contacts personnels, de missions de recherche à l'étranger où l'on peut avoir accès à des centres de documentation bien pourvus.

La somme considérable d'études, de brochures, de dossiers publiés par les chercheurs russes fait l'objet d'une diffusion hasardeuse. Publiés souvent à quelques centaines d'exemplaires, ils sont vendus en dehors de réels circuits de distribution et ne sont pas réellement diffusés dans toute la communauté scientifique. Il faut les saisir au hasard des colloques et des rencontres.

\subsection{L'édition}

L'édition scientifique est aussi en piteux état. Les auteurs ayant des difficultés énormes à trouver de quoi éditer et diffuser largement leurs résultats, on manque aujourd'hui d'ouvrages de référence, de grandes synthèses faites par les géographes les plus féconds. Les éditions universitaires publient, avec de petits moyens, des ouvrages qui sont pour l'essentiel des manuels à l'usage des étudiants. L'édition géographique est confrontée à des problèmes particuliers, dans la mesure où il s'y publie beaucoup de cartes, ce qui exige une très bonne qualité graphique et, de façon presque obligatoire, l'usage de la couleur. Le coût prohibitif de ce type d'édition fait que des résultats remarquables restent dans les cartons ou sont publiés de façon très partielle dans les revues. L'Atlas de la Russie et des pays proches édité en 1995 en France (Brunet, Eckert, Kolossov) n'a ainsi pas eu son 
équivalent en russe, alors que tout le matériau était disponible pour publication. Depuis plusieurs années, un Atlas de l'environnement préparé par les géographes de l'université Lomonossov attend son improbable financement.

Les ouvrages généraux disponibles sont relativement médiocres. Ils résultent de la réédition d'ouvrages anciens ou de l'édition sans véritable renouvellement scientifique de cartes issues de la tradition soviétique. C'est notamment le cas de tous les Atlas régionaux publiés par l'Institut de Topographie et de Géodésie, où une cartographie plus que traditionnelle s'allie à une information environnementale et socio-économique qui n'est pas renouvelée. À côté de cela, un travail de recherche régionale remarquable et innovant comme l'Atlas géopolitique du Caucase ne trouve à être publié qu'à l'INALCO... à Paris.

Les supports d'expression les plus stables restent les grandes revues de référence (Vestnik Moskovskogo Gosudarstvennogo Universiteta, Izvestia Vserossiskogo Geografitcheskogo Obchtchestva, Izvestia Akademii Nauk seria geografitcheskaïa), mais dont le rythme de publication est très lent, ou bien les revues étrangères, qui accueillent les contributions des meilleurs géographes russes. On observe donc une certaine «expatriation » de la publication des résultats. Pourtant, des revues apparaissent çà et là. Elles sont malheureusement souvent éphémères et chercher à se les procurer est un sport mouvementé à l'issue incertaine.

\section{La question des données}

\subsection{Les indicateurs statistiques}

Par rapport à la misère statistique de l'époque soviétique, où les données socio-économiques étaient très rarement publiées, la période actuelle est caractérisée par une très grande abondance d'informations. L'Office Fédéral de la Statistique (GosKomStat) publie toutes sortes d'annuaires qui décrivent de manière très complète la situation des régions. On trouve facilement des chiffres sur la délinquance constatée, les migrations, la situation économique, etc. Il faut néanmoins sérieusement nuancer ce beau paysage statistique. L'avantage de l'ouverture des sources est tempéré par la dégradation progressive de la qualité des indicateurs.

\section{La statistique économique}

L'évolution réelle de l'économie est, généralement, très mal prise en compte par l'appareil statistique. L'économie de l'ombre contribue, aux dires des officiels, pour $25 \%$ au moins à la production nationale de richesse. La validité des données du Produit Intérieur fait ainsi l'objet de très vives discussions. De même, on sait très mal quels sont les effectifs réels des entreprises. Le chômage déguisé est considérable (beaucoup de travailleurs encore portés sur les rôles des entreprises et considérés comme « en congé », sont sans emploi ou travaillent en fait ailleurs). Le nombre de salariés par entreprise est très largement fictif et invérifiable. Il en est de même pour le montant réel des salaires distribués : les enveloppes non déclarées ou les avantages en nature venant en sus du salaire officiel sont monnaie courante. Tout cela complique considérablement, pour tous les 
chercheurs, l'appréhension de la situation sociale et économique. Certes, les statisticiens proposent, grâce à de savantes corrections, des versions améliorées des grands chiffres macro-économiques. Mais ces corrections ne sont proposées que pour certaines échelles (Fédération, grandes régions économiques, régions).

\section{La statistique sociale et démographique}

On a souvent dit que la statistique démographique était par contre d'assez bonne qualité. L'enregistrement de l'état-civil fonctionne, le mouvement naturel est saisi. Bon an mal an, des données de population actualisées, d'échelle fine, sont publiées par le GosKomStat. Mais cela laisse entière la question des transformations sociales. Les formidables mutations des activités de la population, du mode de vie, sont difficiles à saisir, car le GosKomStat ne fournit que de grossières estimations. On attendait avec impatience le nouveau recensement, prévu pour 1999 (le précédent, effectué sous l'U.R.S.S., datait de 1989), qui devait donner de précieuses indications sur les changements sociaux, ethniques, permettre d'apprécier le résultat des mouvements de population. Or ce recensement a été repoussé sine die, le gouvernement fédéral étant dans l'impossibilité de le financer. L'ensemble des données qui devaient être actualisées à l'occasion du recensement va continuer, pendant un temps indéterminé, à faire au mieux l'objet d'estimations annuelles de plus en plus risquées. Les données sur la composition de la population par nationalité sont toujours fournies pour 1989, tout comme celles sur le niveau de diplômes, etc.

\subsection{Le problème des échelles fines}

L'abondance des données régionales a permis de réaliser un très grand nombre de cartes analytiques et synthétiques sur l'état des régions, de concevoir des indices synthétiques de développement, de la situation sociale..., bref de procéder à toutes sortes de typologies des régions. Les annuaires statistiques régionaux publiés avec régularité sont des exemples de bonne présentation : tableaux complets, valeurs existantes pour chacune des 89 régions $^{6}$.

\section{Vers le niveau infrarégional}

Mais ce niveau reste relativement frustrant pour le géographe : les régions russes sont très fortement hétérogènes (superficie, population), on met sur le même plan la gigantesque république de Sakha (ex-Iakoutie, 3 millions de $\mathrm{km}^{2}$ ) avec la ville de Moscou. Les unités statistiques régionales sont en fait des unités administratives dont la cohérence reste à démontrer. Il existe même des enclaves, la plus bizarre étant celle de la ville de Norilsk en territoire Taïmyr, rattachée administrativement à la région de Krasnoïarsk, située bien plus au sud. Les tentatives de cartographie à l'échelle des régions sont biaisées, portent à surestimer à la lecture les zones les moins peuplées du territoire (Sibérie et Extrême-Orient). Tout plaiderait donc pour que l'on cherche à mesurer les phénomènes à l'échelle infrarégionale (Eckert 1996). Le changement d'échelle permet en effet de renouveler et d'affiner les analyses. L'exemple remarquable de l'Atlas 
géopolitique du Caucase montre à quel point les problématiques sont renouvelées par ce saut d'échelle.

Malheureusement, on constate que le paysage statistique s'appauvrit rapidement quand on va vers les échelles fines. Les données existant sur les 2500 districts (raïons) et villes à statut autonome sont très peu nombreuses : sont publiées chaque année les estimations de population rurale et urbaine pour ces unités ; quelques indicateurs datant du recensement de 1989 sont également disponibles (niveau de formation de la population adulte). Si l'on cherche à avoir des données socio-économiques, on s'aperçoit que les tableaux fournis présentent des trous inexpliqués : ici ou là telle donnée manque pour la région de Sverdlovsk, telle autre pour Stavropol, etc. Les bases de données urbaines maintenues par le GosKomStat souffrent des mêmes lacunes. La base dite « 555 » sur les villes est surtout remarquable par les trous qu'elle comporte : certaines villes sont oubliées, certaines variables sont présentes pour une ville mais pas pour une autre, etc. au point qu'on pourrait croire ces tableaux constitués par on ne sait quel statisticien névrosé et capricieux. On sait en réalité que les offices régionaux de la statistique ne transmettent que très partiellement leurs données détaillées au centre fédéral. Dans certaines régions on pourra se procurer telle ou telle donnée qui sera indisponible ailleurs : le résultat est qu'il y a très peu d'ensembles cohérents permettant de décrire à cette échelle décisive l'évolution du pays tout entier.

Il est également évident que les contrôles de cohérence de la saisie ne sont pas faits correctement : pourquoi, dans un tableau que nous avons consulté, dans la ville de Pereslavl-Zalesski (région de Iaroslavl), l'industrie affiche-t-elle, bon an mal an, environ 53000 employés alors qu'il n'y a que 43000 habitants?

\section{Études diachroniques : le casse-tête}

Les données posent des problèmes encore plus redoutables si l'on cherche à mesurer et cartographier les évolutions dans le temps. Lorsque les limites administratives changent, les annuaires statistiques publient les nouvelles valeurs sans prévenir le lecteur que telle ou telle unité a été amputée d'une partie de son territoire! La statistique publique n'assure pas la cohérence spatiale de son information, On est bien en peine de réaffecter correctement les populations concernées par ces changements. Arkhangelsk a perdu 36600 habitants de 1994 à 1995, du simple fait qu'une ville satellite (Mirny) a fait administrativement « sécession ». Entre 1994 et 1995, la plupart des villes «fermées » (villes secrètes du complexe militaro-industriel) sur lesquelles aucune donnée n'était publiée - même leur localisation était tenue cachée - ont été réintégrées dans la statistique. Leurs habitants, qui étaient autrefois comptés avec ceux d'autres villes (beaucoup à Moscou), sont enfin pris en compte au lieu réel de leur résidence. Ce progrès, cette ouverture posent en fait d'énormes problèmes pour les études diachroniques, car les annuaires ne donnent aucune clé de passage d'une année sur l'autre pour ces unités urbaines soudain reconnues. Les problèmes de cartographie diachronique deviennent alors insolubles. 
De ce fait, la géographie dite "quantitative », qui base ses analyses sur le traitement de données statistiques, est souvent critiquée, dans la mesure où les données disponibles n'ont pas le bel ordonnancement qui permettrait au chercheur de les manipuler sereinement. Le divorce entre le pays réel et le pays décrit par la statistique publique est tel que des contestations radicales sont apparues. Le débat est lancé dans les milieux scientifiques : ne faut-il pas provisoirement répudier l'approche statistique, et décrire les changements par des méthodes qualitatives, en multipliant le travail de terrain, les entretiens, sans trop s'intéresser aux données? La polémique n'est pas close.

\subsection{Comment accède-t-on à l'information?}

Il est, nous l'avons vu, assez difficile de se procurer pour le pays de grands ensembles de données cohérents : les trous et les imperfections abondent. La plupart du temps, l'information disponible par districts et par villes n'est pas disponible pour tout le pays. Le travail de collecte des données est plus facile si l'on se restreint à une seule région, mais on limite le champ d'analyse. Par ailleurs, pour qui s'intéresse à l'échelle du local et de l'intra-urbain, de nombreuses informations pertinentes sur l'évolution réelle des économies urbaines (implantation des commerces, marché foncier, zones d'activité) sont produites et maintenues par les municipalités à fins de gestion. Or les pouvoirs locaux gèrent au cas par cas l'accès à ces données, et le succès dépend du réseau d'accès à l'information qu'aura su constituer le chercheur (« sur la base de contacts personnels », comme on dit joliment en russe). La municipalité de Moscou, qui dispose de bases de données solidement organisées sur l'évolution de l'espace urbain (population, quartiers, activités, fiscalité, marché foncier) ne les rend accessibles qu'aux équipes qui acceptent de réaliser des études pour elle. On n'est donc pas dans le cadre d'une information publiée et accessible à tous.

\section{Cartographie et systèmes d'information géographiques}

Parallèlement à tous les champs de recherche mentionnés, il faut signaler le remarquable progrès des techniques cartographiques et le recours de plus en plus systématique à la cartomatique, qui se manifeste notamment par l'édition de superbes plans de villes (plusieurs d'entre d'eux repèrent tous les immeubles de Moscou) qui relèguent le récent passé soviétique à une sorte de préhistoire cartographique (Eckert 1998).

Les municipalités et les organismes gestionnaires les plus en pointe se sont lancés dans l'implémentation de systèmes d'information géographiques performants (cadastre, police) et ont largement numérisé leurs cartes de base. L'exemple est surtout donné par Moscou, mais partout où l'on a les moyens, on cherche à suivre le mouvement. Le marché de ces technologies géographiques n'est pas totalement dominé par les produits logiciels étrangers, puisque des centres de ressources russes ont su développer leurs propres produits et les commercialiser avec succès? 


\section{Conclusion}

La géographie humaine, à l'image de la Russie, offre un paysage turbulent et brouillé. Elle est en même temps active et attentive aux évolutions du pays. Mais sa capacité d'analyse est amoindrie par le manque de données homogènes et cohérentes, par la nécessité d'aller vite pour produire des résultats. La recherche sous contrat, dite « alimentaire », est très fréquente et parfois féconde, mais elle n'encourage pas la formulation de projets et de problématiques sur la moyenne et la longue durée. Il en est de même des bourses et subventions accordées par les organismes nationaux et internationaux : les horizons sont en général d'un ou deux ans, après quoi il faut déposer d'autres projets, se démarquant de préférence des projets immédiatement antérieurs. La situation est certes sérieuse, les manques et les frustrations certains : il n'en faut tirer ni raisons de désespérer, ni celles de succomber à un optimisme béat.

\section{NOTES}

1. Rossijskij Fond Fundamental'nyx Issledovanij.

2. Ce fut notamment le cas de la mise en œuvre des Complexes Territoriaux de Production dans les années 1950-60 sous l'influence de Kolossovski.

3. Le laborieux pape de la géographie industrielle, l'octogénaire Khrouchtchev en est le meilleur exemple.

4. INTAS 96-0069 «Evaluation of the investment climate and of the Socio-Economic Conditions in Russian Regions »

5. L'Espace géographique parvient à la bibliothèque de l'Institut de Géographie de l'Académie des Sciences à Moscou, mais grâce à un accord particulier d'échange avec la publication de cet Institut (Izvestija Akademii Nauk, serija geografiðeskaja). Ce type d'accord ponctuel est toujours fragile et résulte du hasard des contacts établis entre institutions. Ils ne peuvent se substituer à une réelle politique documentaire.

6. Sauf la Tchétchénie, pour laquelle on ne dispose pratiquement plus d'information statistique depuis la guerre de 1994-1996.

7. On pense particulièrement aux logiciels Geodraw et GeoGraph développés par le Centre des Systèmes d'Information Géographique de I'Institut de Géographie de l'Académie des Sciences (Moscou). 


\section{Références :}

Sur l'évolution des problématiques et les problèmes méthodologiques de la géographie russe:

Eckert D. (1998), « Progrès de la cartographie et pratique de la ville: Moscou ou la culture de la désorientation », Mappemonde, $\mathrm{n}^{\circ}$ 51, p. 2326.

Eckert D. (1996), «Des régions aux districts en Russie : nouvelles échelles d'analyse du territoire ", Mappemonde, $\mathrm{n}^{\circ} 3, \mathrm{p} .1-8$.

Eckert D., Treïvich A. (1995), « La géographie industrielle, discipline en crise dans la recherche russe », L'Espace Géographique, n² 2, p. 169180.

Gritsai O. (1998), "Russian Human Geography in the Wonderland of Globalization ", Geojournal, 45.1-2 : 77-83.

Колосов В. А., Мироненко Н. С., Петров Н. В., Трейвиш А. И., « Теория и социальные функции географии», Известия Академии Наук, серия географическая, $\mathrm{n}^{\circ} 3,1996,7-22$.

Sur l'évolution du territoire russe :

Béroutchachvili N., Radvanyi J. (dir.), 1996, Atlas Géopolitique informatique du Caucase, Paris, INALCO, classeur avec feuilles volantes, non paginé.

Brunet R. (1996), «La Russie et les pays proches » in Géographie Universelle, t. 10, Belin-Reclus.

Brunet R., Eckert D., Kolossov V., dir. (1995), Atlas de la Russie et des pays proches, Montpellier-Paris : Reclus - La Documentation française, $208 \mathrm{p}$.

Collectif (1997), « Moscow and St Petersburg in Transition », $\mathrm{n}^{\circ}$ spécial de Geojournal, vol. $42 \mathrm{n}^{\circ} 4$, août 1997.

Eckert D., Kolossov V. (1999), La Russie, Flammarion, coll. Dominos, $128 \mathrm{p}$.

Kolossov V., Platé A. (1997), «la Russie partagée : géographie des élections ", Mappemonde, 1997, $\mathrm{n}^{\circ}$ 3, p. 14-19.

Treivish A. (1998), "Kostroma Oblast' : An Average-Russian, Averagelydepressed Region ", Communist Economics \& Economic Transformation, Vol. 10, $\mathrm{n}^{\circ} 3$

\section{Autres références}

Huntington S. (1997), Le choc des civilisations, O. Jacob, 402 p. publié en version originale anglaise en 1996 sous le titre The Clash of Civilizations and the Remaking of World Order. 